Cahiers $d u$ MONDE RUSSE

\section{Cahiers du monde russe}

Russie - Empire russe - Union soviétique et États indépendants

$55 / 3-4 \mid 2014$

Varia

\title{
Nancy Kollmann, Crime and Punishment in Early Modern Russia
}

\section{Дмитрий Серов}

\section{(2) OpenEdition}

\section{Journals}

Édition électronique

URL : http://journals.openedition.org/monderusse/8020

DOI : $10.4000 /$ monderusse. 8020

ISSN : $1777-5388$

\section{Éditeur}

Éditions de l'EHESS

\section{Édition imprimée}

Date de publication : 1 juillet 2014

Pagination : $345-348$

ISBN : 978-2-7132-2441-6

ISSN : $1252-6576$

\section{Référence électronique}

Дмитрий Серов, « Nancy Kollmann, Crime and Punishment in Early Modern Russia », Cahiers du monde russe [Онлайн], 55/3-4 | 2014, Выложить онлайн 08 avril 2015, Наводить справки в 25 septembre 2020. URL : http://journals.openedition.org/monderusse/8020 ; DOI : https://doi.org/ 10.4000/monderusse. 8020

Ce document a été généré automatiquement le 25 septembre 2020.

(c) École des hautes études en sciences sociales 


\title{
Nancy Kollmann, Crime and Punishment in Early Modern Russia
}

\author{
Дмитрий Серов
}

\section{RÉFÉRENCE}

Nancy KOLLMANN, Crime and Punishment in Early Modern Russia, Cambridge :

Cambridge University Press, 2012, xvi +488 p.

1 ВЫХОД В СВЕТ МОНОГРАФИИ НЭНСИ КОЛЛМАНН « ПРЕСТУПЛЕНИЕ И НАКАЗАНИЕ В РОССИИ РАННЕГО НОВОГО ВРЕМЕНИ» НЕОБХОДИМО ПРИЗНАТЬ ГЛУБОКО ОТРАДНЫМ СОБЫТИЕМ ДЛЯ ВСЕХ, КТО УГЛУБЛЕННО ИНТЕРЕСУЕТСЯ ОТДАЛЕННЫМ ИСТОРИЧЕСКИМ ПРОШЛЫМ ЭТОЙ СТРАНЫ. ПРЕДСТАВЛЯЕТСЯ ОЧЕВИДНЫМ, ЧТО В ДАННОМ СЛУЧАЕ НЭНСИ КОЛЛМАНН ПРОДОЛЖИЛА ИСТОРИОГРАФИЧЕСКУЮ ЛИНИЮ, ОПРЕДЕЛЕННО НАМЕЧЕННУЮ МОСКОВСКИМ ПРАВОВЕДОМ В.А. РОГОВЫМ (1947-2009), ИЗДАВШИМ В 1995 Г. ДИССЕРТАЦИОННУЮ МОНОГРАФИЮ « ИСТОРИЯ УГОЛОВНОГО ПРАВА, ТЕРРОРА И РЕПРЕССИЙ В РУССКОМ ГОСУДАРСТВЕ XV-XVII ВВ.» ${ }^{1}$. ОДНАКО НАЗВАННАЯ РАБОТА ВЛАДИМИРА РОГОВА НЕ ТОЛЬКО ОХВАТЫВАЕТ БОЛЕЕ УЗКИЙ ХРОНОЛОГИЧЕСКИЙ ПЕРИОД, НО И НЕОСПОРИМО УСТУПАЕТ НОВОЙ КНИГЕ Н. КОЛЛМАНН КАК ПО ШИРОТЕ ПРОБЛЕМАТИКИ И ОБЪЕМУ ПРИВЛЕЧЕННЫХ ИСТОЧНИКОВ (ОСОБЕННО АРХИВНЫХ), ТАК И ПО СИСТЕМАТИЧНОСТИ АНАЛИЗА ОПИСЫВАЕМЫХ СОБЫТИЙ И ЯВЛЕНИЙ.

2 В ЭТОМ ОТНОШЕНИИ ОСТАЕТСЯ ТОЛЬКО НЕДОУМЕВАТЬ, ОТЧЕГО ЗА АКАДЕМИЧЕСКИ ТЩАТЕЛЬНУЮ РАЗРАБОТКУ ТЕМЫ, ИЗБРАННОЙ Н. КОЛЛМАНН, НЕ ВЗЯЛСЯ НИКТО ИЗ РОССИЙСКИХ ИССЛЕДОВАТЕЛЕЙ, В ЧАСТНОСТИ НИ ОДИН ПРЕДСТАВИТЕЛЬ МНОГОЧИСЛЕННЫХ КАФЕДР ТЕОРИИ И ИСТОРИИ ГОСУДАРСТВА И ПРАВА ВЫСШИХ УЧЕБНЫХ ЗАВЕДЕНИЙ ЮРИДИЧЕСКОГО ПРОФИЛЯ. НЕЛЬЗЯ ТАКЖЕ НЕ ВЫРАЗИТЬ СОЖАЛЕНИЯ, ЧТО В ОПУБЛИКОВАННОЙ УЖЕ В 2014 Г. ДВУХТОМНОЙ МОНОГРАФИИ А.В. НАУМОВА «ПРЕСТУПЛЕНИЕ И НАКАЗАНИЕ В ИСТОРИИ РОССИИ» РАЗДЕЛ, ПОСВЯЩЕННЫЙ XV - ПЕРВОЙ ЧЕТВЕРТИ XVIII ВВ., ЯВИЛ СОБОЙ КРАЙНЕ 
ПОВЕРХНОСТНЫЙ И ПРИ ТОМ ФРАГМЕНТАРНЫЙ ОБЗОР РАЗВИТИЯ УГОЛОВНОГО ЗАКОНОДАТЕЛЬСТВА ОТМЕЧЕННОГО ПЕРИОДА 2 .

ПЕРЕХОДЯ К ХАРАКТЕРИСТИКЕ ТРУДА Н. КОЛЛМАНН, ДЛЯ НАЧАЛА ВИДИТСЯ УМЕСТНЫМ ОТМЕТИТЬ, ЧТО АВТОР СУМЕЛА ВЫЯВИТЬ ПОЧТИ ИСЧЕРПЫВАЮЩИЙ КРУГ РАБОТ ПРЕДШЕСТВЕННИКОВ И ПУБЛИКАЦИЙ ДОКУМЕНТАЛЬНЫХ И ПОВЕСТВОВАТЕЛЬНЫХ ИСТОЧНИКОВ, ИМЕЮЩИХ ДАЖЕ КОСВЕННОЕ ОТНОШЕНИЕ К ТЕМЕ ИССЛЕДОВАНИЯ. НЕЛЬЗЯ НЕ ВПЕЧАТЛИТЬСЯ И КОЛИЧЕСТВОМ ИСПОЛЬЗОВАННЫХ НЭНСИ КОЛЛМАНН АРХИВНЫХ ДОКУМЕНТОВ, ИЗВЛЕЧЕННЫХ ИЗ 54 ( !) ФОНДОВ РГАДА. В КАЧЕСТВЕ ДОПОЛНЕНИЯ К ФУНДАМЕНТАЛЬНОЙ «БИБЛИОГРАФИИ» (с. 458-478) ВОЗМОЖНО УКАЗАТЬ РАЗВЕ ЧТО НА ПРОСТРАННУЮ СТАТЬЮ В.И. КУРДИНОВСКОГО 1895 Г. О ГУБНЫХ ИЗБАХ, НА ОБШИРНУЮ СТАТЬЮ П.С. РОМАШКИНА 1949 Г. ОБ УГОЛОВНОМ ЗАКОНОДАТЕЛЬСТВЕ ПЕТРАІ, НА СТАТЬЮ Н.Б. ГОЛИКОВОЙ 1991 Г. ОБ ОРГАНИЗАЦИИ БОРЬБЫ С ГОСУДАРСТВЕННЫМИ ПРЕСТУПЛЕНИЯМИ В XVI-XVII ВВ. ДА НА НЕДАВНЮЮ МОНОГРАФИЮ П. ХОФФМАННА «ПЕТР ВЕЛИКИЙ КАК ВОЕННЫЙ РЕФОРМАТОР И ПОЛКОВОДЕЦ», В КОТОРОЙ МЕЖДУ ИНОГО ОКАЗАЛИСЬ ЗАТРОНУТЫ ВОПРОСЫ ИСТОРИИ ВОЕННО-УГОЛОВНОГО И ВОЕННО-ПРОЦЕССУАЛЬНОГО ЗАКОНОДАТЕЛЬСТВА РОССИИ ПЕРВОЙ ЧЕТВЕРТИ XVIII В. ${ }^{3}$

ОПИРАЯСЬ НА ОБРАЗЦОВО ПРОЧНУЮ ИСТОРИОГРАФИЧЕСКУЮ И ИСТОЧНИКОВУЮ ОСНОВУ, Н. КОЛЛМАНН ПОДГОТОВИЛА БЕССПОРНО ВЫДАЮЩЕЕСЯ, НЕ ИМЕЮЩЕЕ НА СЕГОДНЯ АНАЛОГОВ ИССЛЕДОВАНИЕ ПО ИСТОРИИ РАЗВИТИЯ УГОЛОВНОГО ПРАВА И УГОЛОВНОГО ПРОЦЕССА В РОССИИ КОНЦА XV - ПЕРВОЙ ЧЕТВЕРТИ XVIII ВВ. ПРИНЦИПИАЛЬНО ВАЖНЫМ ДОСТОИНСТВОМ ИЗЫСКАНИЙ АВТОРА СЛЕДУЕТ ПРИЗНАТЬ ТО ОБСТОЯТЕЛЬСТВО, ЧТО ОНА НЕ ОСТАНОВИЛАСЬ НА ОБОЗРЕНИИ НОРМАТИВНОГО МАТЕРИАЛА (ЧЕМ, КАК ПРАВИЛО, ОГРАНИЧИВАЮТСЯ ИСТОРИКИ ПРАВА ${ }^{4}$ ), А ЦЕЛЕНАПРАВЛЕННО И МАКСИМАЛЬНО ШИРОКО ПРИВЛЕКЛА ПРАВОПРИМЕНИТЕЛЬНЫЕ АКТЫ. БЛАГОДАРЯ ПОДОБНОМУ ПОДХОДУ, Н. КОЛЛМАНН УДАЛОСЬ ПРЕВРАТИТЬ МОНОГРАФИЮ В СВОЕГО РОДА ИСТОРИОГРАФИЧЕСКУЮ ГОЛОГРАММУ, В ПОЛНОЙ МЕРЕ РАСКРЫВ ИЗБРАННУЮ ТЕМУ. В ЭТОМ ОТНОШЕНИИ, ДУМАЕТСЯ, ОСОБЕННЫЙ ИНТЕРЕС ДЛЯ ЧИТАТЕЛЯ ПРЕДСТАВЯТ В ЧАСТНОСТИ ТАКИЕ ПАРАГРАФЫ МОНОГРАФИИ КАК « ВОЕВОДА И ГУБНОЙ СТАРОСТА НА БЕЛООЗЕРЕ И В АРЗАМАСЕ» (С. 35-36), « ПРАКТИКА ВЫНЕСЕНИЯ СУДЕБНЫХ РЕШЕНИЙ» (С. 160-165), «ПЕТРОВСКИЕ СУДЬИ ЗА РАБОТОЙ» (С. 191-194), « ПРАКТИКА НАКАЗАНИЙ ДО 1649 Г.» (С. 215-222), « ЗАКОН В ДЕЙСТВИИ В APЗAMACE» (C. 267-279).

5 В СВОЕМ ОБРАЩЕНИИ К ИСТОРИИ УГОЛОВНОГО ПРАВА НЭНСИ КОЛЛМАНН УДЕЛИЛА ПРИОРИТЕТНОЕ ВНИМАНИЕ РАССМОТРЕНИЮ СИСТЕМЫ УГОЛОВНО-ПРАВОВЫХ САНКЦИЙ, ЧЕМУ ОКАЗАЛАСЬ ПОСВЯЩЕНА ОДНА ИЗ ДВУХ ЧАСТЕЙ КНИГИ (ЧАСТЬ 2 «НАКАЗАНИЕ», ГЛАВЫ 9-18). ЗДЕСЬ АВТОР СУМЕЛА ВОССОЗДАТЬ УНИКАЛЬНУЮ ПО ФАКТОЛОГИЧЕСКОЙ НАСЫЩЕННОСТИ КАРТИНУ УГОЛОВНЫХ РЕПРЕССИЙ В РОССИИ КОНЦА XV - ПЕРВОЙ ЧЕТВЕРТИ XVIII ВВ. Н. КОЛЛМАНН СИСТЕМАТИЧЕСКИ ОСВЕТИЛА КАК НОРМАТИВНОЕ РЕГУЛИРОВАНИЕ РАЗЛИЧНЫХ ВИДОВ НАКАЗАНИЙ (ОТ СМЕРТНОЙ КАЗНИ ДО ССЫЛКИ), ТАК И ПРАКТИКУ НАЗНАЧЕНИЯ ЭТИХ ВИДОВ НАКАЗАНИЙ НА ВСЕМ ПРОТЯЖЕНИИ ОПИСЫВАЕМОГО ИСТОРИЧЕСКОГО ПЕРИОДА. НАИБОЛЕЕ ПРИМЕЧАТЕЛЬНЫМИ В ДАННОЙ ЧАСТИ ПРЕДСТАВЛЯЮТСЯ ГЛАВЫ 10 «ТЕЛЕСНЫЕ НАКАЗАНИЯ В 1649-1698 ГГ.» (С. 223-240), 13 « СМЕРТНАЯ КАЗНЬ : ВИДЫ И РИТУАЛЫ» (с. 280-302) И 15 « МЯТЕЖ И ВОССТАНИЕ» (С. 356-379). 
ЧТО КАСАЕТСЯ РАЗДЕЛА МОНОГРАФИИ, ПОСВЯЩЕННОГО УГОЛОВНОМУ ПРОЦЕССУ, ТО ЗДЕСЬ НЭНСИ КОЛЛМАНН ПОДРОБНО ОСТАНОВИЛАСЬ НА ВОПРОСАХ О СИСТЕМЕ ДОКАЗАТЕЛЬСТВ (С. 114-127), О ПОРЯДКЕ ВЫНЕСЕНИЯ, ПЕРЕСМОТРА И УТВЕРЖДЕНИЯ ПРИГОВОРОВ И ПРАКТИКЕ ПОМИЛОВАНИЙ (С. 160-176), А ТАКЖЕ УГЛУБЛЕННО ПРОАНАЛИЗИРОВАЛА ПРОБЛЕМУ ИСПОЛЬЗОВАНИЯ В ТОГДАШНЕМ РОССИЙСКОМ СУДОПРОИЗВОДСТВЕ ПЫТОК (С. 133-156). В ДАННОМ СЛУЧАЕ НЕДОУМЕНИЕ ВЫЗЫВАЕТ ЕДИНСТВЕННО ТО ОБСТОЯТЕЛЬСТВО, ЧТО АВТОР НИ СЛОВОМ НЕ УПОМЯНУЛА О ПОДРОБНОЙ РЕГЛАМЕНТАЦИИ ПРИМЕНЕНИЯ ПЫТОК В ТАКОМ ИЗВЕСТНОМ И НЕОДНОКРАТНО ПУБЛИКОВАВШЕМСЯ ЗАКОНОДАТЕЛЬНОМ АКТЕ КАК «КРАТКОЕ ИЗОБРАЖЕНИЕ ПРОЦЕССОВ ИЛИ СУДЕБНЫХ ТЯЖЕБ» 1715 Г. . КРОМЕ ТОГО, К ДАННЫМ, ПРИВЕДЕННЫМ Н. КОЛЛМАНН, ПРЕДСТАВЛЯЕТСЯ УМЕСТНЫМ ДОБАВИТЬ, ЧТО В НАКАЗЕ « МАЙОРСКИМ» СЛЕДСТВЕННЫМ КАНЦЕЛЯРИЯМ ОТ 9 ДЕКАБРЯ 1717 Г. ОКАЗАЛСЯ ВПЕРВЫЕ НОРМАТИВНО ЗАКРЕПЛЕН ОСОБЫЙ ПОРЯДОК НАЗНАЧЕНИЯ ПЫТОК СТРОЕВЫМ ОФИЦЕРАМ, А ТАКЖЕ ВЫСОКОПОСТАВЛЕННЫМ ГОСУДАРСТВЕННЫМ ГРАЖДАНСКИМ СЛУЖАЩИМ. СОГЛАСНО ОТМЕЧЕННОМУ НАКАЗУ, ПРИМЕНЕНИЕ ПЫТКИ В ОТНОШЕНИИ МЛАДШИХ ОФИЦЕРОВ ДОЛЖНО БЫЛО САНКЦИОНИРОВАТЬСЯ ВОЕННЫМ СУДОМ (!), А В ОТНОШЕНИИ СТАРШИХ И ВЫСШИХ ОФИЦЕРОВ И ДОЛЖНОСТНЫХ ЛИЦ ГРАЖДАНСКОЙ АДМИНИСТРАЦИИ ОТ ВИЦЕ-ГУБЕРНАТОРА И ВЫШЕ - САМИМ МОНАРХОМ ${ }^{6}$.

7 ЗНАЧИТЕЛЬНЫЙ ИНТЕРЕС ПРЕДСТАВЛЯЮТ ИЗЛОЖЕННЫЕ В ГЛАВЕ 2 МОНОГРАФИИ (С. 48-65) МНОГООБРАЗНЫЕ СВЕДЕНИЯ И НАБЛЮДЕНИЯ Н. КОЛЛМАНН О КВАЛИФИКАЦИОННОМ УРОВНЕ КАНЦЕЛЯРСКИХ СЛУЖАЩИХ, СВЯЗАННЫХ С СУДОПРОИЗВОДСТВОМ, А ТАКЖЕ О КОРПОРАЦИИ ПЛОЩАДНЫХ ПОДЬЯЧИХ (town-square scribes). ЗДЕСЬ АВТОР ВЫШЛА НА ВАЖНУЮ (И ДОНЫНЕ МАЛОРАЗРАБОТАННУЮ) ПРОБЛЕМУ СПЕЦИФИКИ ПРОФЕССИОНАЛЬНОЙ ПОДГОТОВЛЕННОСТИ РОССИЙСКОГО ПРИКАЗНОГО ПЕРСОНАЛА ХVI-XVII ВВ. СПЕЦИФИКА ЭТА (НЕ ВПОЛНЕ, ПРАВДА, ОСОЗНАННАЯ НЭНСИ КОЛЛМАНН) ЗАКЛЮЧАЛАСЬ В ТОМ, ЧТО, НЕ ИМЕЯ ВОЗМОЖНОСТИ ПОЛУЧИТЬ СИСТЕМАТИЧЕСКОЕ ШКОЛЬНОЕ И АКАДЕМИЧЕСКОЕ ОБРАЗОВАНИЕ, ТОГДАШНИЕ ДЬЯКИ И ПОДЬЯЧИЕ С ЛИХВОЙ КОМПЕНСИРОВАЛИ ОТСУТСТВИЕ ТЕОРЕТИЧЕСКОЙ ПОДГОТОВКИ ОБШИРНОСТЬЮ ПРИКЛАДНЫХ ЗНАНИЙ. ТЕМ САМЫМ, ЮРИДИЧЕСКУЮ НЕИСКУШЕННОСТЬ ПРИКАЗНОГО СУДЬИ, ВОЕВОДЫ ИЛИ ГУБНОГО СТАРОСТЫ УСПЕШНО ВОСПОЛНЯЛ СОСТОЯВШИЙ ПРИ НЕМ ПРАКТИЧЕСКИЙ ЗАКОНОВЕД В ЛИЦЕ КАНЦЕЛЯРСКОГО СЛУЖИТЕЛЯ.

СИСТЕМАТИЧЕСКИ ОБОЗРЕВАЯ РАЗВИТИЕ УГОЛОВНОГО ПРАВА РОССИИ КОНЦА ХV ПЕРВОЙ ЧЕТВЕРТИ ХVIII ВВ., Н. КОЛЛМАНН НЕ МОГЛА, РАЗУМЕЕТСЯ, НЕ ЗАТРОНУТЬ И ПРОБЛЕМУ ВЗЯТОЧНИЧЕСТВА, ПОБОРОВ С НАСЕЛЕНИЯ СО СТОРОНЫ ДОЛЖНОСТНЫХ ЛИЦ ВСЕХ УРОВНЕЙ. ОЗНАЧЕННОЙ ПРОБЛЕМЕ ОКАЗАЛИСЬ СПЕЦИАЛЬНО ПОСВЯЩЕНЫ (ПРИМЕНИТЕЛЬНО К РАЗНЫМ ПЕРИОДАМ) ОДНА ИЗ ГЛАВ И ПАРАГРАФ МОНОГРАФИИ (С. 93-112, 194-196). НА УКАЗАННЫХ СТРАНИЦАХ АВТОР ПРИВЕЛА ИНТЕРЕСНЕЙШИЕ ДАННЫЕ О МАСШТАБАХ РАСПРОСТРАНЕННОСТИ ВЗЯТОЧНИЧЕСТВА, О ПРАВИТЕЛЬСТВЕННЫХ МЕРАХ ПО ЕГО ОБУЗДАНИЮ, ОБ УГОЛОВНОМ ПРЕСЛЕДОВАНИИ ВЗЯТОЧНИКОВ. К ИЗЛОЖЕННОМУ В МОНОГРАФИИ СТОИТ ДОБАВИТЬ, ЧТО В ХVII В. ПОЗИЦИЯ РОССИЙСКОГО ЗАКОНОДАТЕЛЯ В ОТНОШЕНИИ ПОБОРОВ С НАСЕЛЕНИЯ ОТЛИЧАЛАСЬ В ЦЕЛОМ НЕПОСЛЕДОВАТЕЛЬНОСТЬЮ. ДОСТАТОЧНО ВСПОМНИТЬ, ЧТО САМО ПО СЕБЕ ПОЛУЧЕНИЕ ВЗЯТКИ (« ПОСУЛА») НЕ ПОДВЕРГЛОСЬ ЗАПРЕЩЕНИЮ В УЛОЖЕНИИ 1649 Г. СОГЛАСНО НОРМАМ, ЗАКРЕПЛЕННЫМ В ГЛАВАХ 7-Й, 10-Й И 21-Й УЛОЖЕНИЯ, ДОЛЖНОСТНЫЕ ЛИЦА ПОДЛЕЖАЛИ УГОЛОВНОЙ ОТВЕТСТВЕННОСТИ 
ТОЛЬКО В ТОМ СЛУЧАЕ, ЕСЛИ ПРИНЯТИЕ ПОСУЛА ОБУСЛОВИЛО СОВЕРШЕНИЕ ИМИ ИНЫХ ПРЕСТУПНЫХ ДЕЯНИЙ ПРОТИВ ИНТЕРЕСОВ СЛУЖБЫ И ПРОТИВ ПРАВОСУДИЯ - ОТ ВЫНЕСЕНИЯ НЕПРАВОСУДНОГО ПРИГОВОРА ДО СЛУЖЕБНОГО ПОДЛОГА? ВЫВОДЫ ПО МОНОГРАФИИ НЭНСИ КОЛЛМАНН СОСРЕДОТОЧИЛА В ЗАКЛЮЧЕНИИ, ОЗАГЛАВЛЕННОМ «РОССИЙСКАЯ ПРАВОВАЯ КУЛЬТУРА» (С. 416-426). ИЗ ЧЕРЕДЫ МНОГИХ ГЛУБОКИХ СУЖДЕНИЙ, СФОРМУЛИРОВАННЫХ В ЗАКЛЮЧЕНИИ, НАИБОЛЕЕ СУЩЕСТВЕННЫМ, ДУМАЕТСЯ, НЕОБХОДИМО ПРИЗНАТЬ СЛЕДУЮЩЕЕ, ОТНОСЯЩЕЕСЯ К СУДЕБНОЙ СИСТЕМЕ РОССИИ XVI-XVII ВВ.: «There are many indications that the system provided justice...» ${ }^{8}$ (с. 418). В ЭТОЙ СВЯЗИ НЕЛЬЗЯ ТАКЖЕ НЕ СОГЛАСИТЬСЯ С МНЕНИЕМ Н. КОЛЛМАНН О НЕОБОСНОВАННОСТИ ТОГО КРИТИЧЕСКОГО ПРЕНЕБРЕЖЕНИЯ, С КОТОРЫМ МНОГИЕ СОВРЕМЕННЫЕ ПРОСВЕЩЕННЫЕ РОССИЯНЕ ОТНОСЯТСЯ К НАЦИОНАЛЬНОМУ ПРАВОВОМУ НАСЛЕДИЮ XV-XVIII ВВ. (С. 426).

О ЧЕМ ХОТЕЛОСЬ БЫ СКАЗАТЬ, ПОДВОДЯ ИТОГ ИЗЛОЖЕННОМУ ВЫШЕ ? НЕСМОТРЯ НА ОТДЕЛЬНЫЕ КОМПОЗИЦИОННЫЕ И СОДЕРЖАТЕЛЬНЫЕ «ШЕРОХОВАТОСТИ» И НЕЗНАЧИТЕЛЬНЫЕ ИСТОЧНИКОВЫЕ УПУЩЕНИЯ, МОНОГРАФИЯ НЭНСИ КОЛЛМАНН « ПРЕСТУПЛЕНИЕ И НАКАЗАНИЕ В РОССИИ РАННЕГО НОВОГО ВРЕМЕНИ» ОБРАЗОВАЛА СОБОЙ ЗНАЧИТЕЛЬНУЮ ВЕХУ В ИЗУЧЕНИИ ИСТОРИИ ГОСУДАРСТВА И ПРАВА РОССИИ XV-XVIII ВВ. ОТНЫНЕ ЛЮБОЙ ИССЛЕДОВАТЕЛЬ, КОТОРЫЙ ВОЗНАМЕРИТСЯ ОБРАТИТЬСЯ К ИСТОРИКО-ПРАВОВОЙ ПРОБЛЕМАТИКЕ ОТМЕЧЕННОГО ПЕРИОДА, ДОЛЖЕН БУДЕТ НАЧАТЬ СВОИ ИЗЫСКАНИЯ $С$ ОЗНАКОМЛЕНИЯ $С$ ЭТИМ ИНТЕРЕСНЕЙШИМ ФУНДАМЕНТАЛЬНЫМ ТРУДОМ.

\section{NOTES}

1. 1 - В.А. РОГОВ, ИСТОРИЯ УГОЛОВНОГО ПРАВА, ТЕРРОРА И РЕПРЕССИЙ В РУССКОМ ГОСУДАРСТВЕ XV-XVII ВВ., М. : ЮРИСТЪ, 1995. СР. : Idem, ПРОБЛЕМЫ ИСТОРИИ РУССКОГО УГОЛОВНОГО ПРАВА, XV - СЕРЕДИНА ХVII ВВ. : ДИСС. ... Д-РА ЮРИД. НАУК, М., 1999.

2. А.В. НАУМОВ, ПРЕСТУПЛЕНИЕ И НАКАЗАНИЕ В ИСТОРИИ РОССИИ, Ч. 1 , М. : ЮРЛИТИНФОРМ, 2014, С. 15-42.

3. В.И. КУРДИНОВСКИЙ, «ГУБНЫЕ УЧРЕЖДЕНИЯ МОСКОВСКОГО ГОСУДАРСТВА», ЖУРНАЛ МИНИСТЕРСТВА НАРОДНОГО ПРОСВЕЩЕНИЯ, № 10, 1895, с. 280-318; № 12, 1895, с. 309-342 ; П.С. РОМАШКИН, «ПРЕСТУПЛЕНИЕ И НАКАЗАНИЕ ПО ЗАКОНОДАТЕЛЬСТВУ ПЕТРА І», УЧЕНЫЕ ЗАПИСКИ МГУ, 144, М.: ИЗД. МГУ, 1949, С. 149-198; Н.Б. ГОЛИКОВА, «ОРГАНИЗАЦИЯ ПОЛИТИЧЕСКОГО СЫСКА В РОССИИ XVI-XVII ВВ.», in Н.Б. ГОЛИКОВА, РЕД., ГОСУДАРСТВЕННЫЕ УчРЕЖДЕНИЯ РОССИИ XVI-XVIII ВВ., М. : ИзД. МГУ, 1991, с. 11-36 ; Р. Hoffmann, Peter der Große als Militärreformer und FEldherr, Francfurt am Main: Peter Lang International Academic Publishing, 2010, S. 159-194.

4. ПОМИМО МНОЖЕСТВА ПРИМЕРОВ ИЗ РАБОТ РОССИЙСКИХ ИССЛЕДОВАТЕЛЕЙ, ВОЗМОЖНО ВСПОМНИТЬ ИЗВЕСТНУЮ МОНОГРАФИЮ К. ШМИДТА «СОЦИАЛЬНЫЙ КОНТРОЛЬ В МОСКВЕ : ПРАВОСУДИЕ, ПРЕСТУПНОСТЬ И КРЕПОСТНОЕ ПРАВО. 1649-1785 ГГ.». РАССМОТРЕВ (С РАЗНОЙ СТЕПЕНЬЮ ДЕТАЛЬНОСТИ) НОРМЫ АРТИКУЛА ВОИНСКОГО 1715 Г. И « КРАТКОГО ИЗОБРАЖЕНИЯ ПРОЦЕСОВ И СУДЕБНЫХ ТЯЖЕБ» 1715 Г., КРИСТОФ ШМИДТ НЕ ПРИВЕЛ ЗАТЕМ НИ ЕДИНОГО 
ЭПИЗОДА ПРИМЕНЕНИЯ ЭТИХ ЗАКОНОДАТЕЛЬНЫХ АКТОВ НА ПРАКТИКЕ (c. Schmidt, Sozialkontrolle in Moskau. Justiz, Kriminalität und Leibeigenschaft : 1649-1785, Stuttgart : F. Steiner Verlag, 1996, S. 139-143).

5. «КРАТКОЕ ИЗОБРАЖЕНИЕ ПРОЦЕСОВ ИЛИ СУДЕБНЫХ ТЯЖЕБ», in А.А. ПРЕОБРАЖЕНСКИЙ, Т.Е. НОВИЦКАЯ, РЕД., ЗАКОНОДАТЕЛЬСТВО ПЕТРА І, М.: ЮРИДИЧЕСКАЯ ЛИТЕРАТУРА, 1997, C. 837-839.

6. «РОЗЫСКАТЬ НАКРЕПКО, ПРАВДОЮ, БЕЗ ВСЯКИХ ПРИКАЗНЫХ КРЮЧКОВ» : УКАЗЫ ПЕТРА І, ЕКАТЕРИНЫ I И СЕНАТА В ОБЛАСТИ СУДОУСТРОЙСТВА И УГОЛОВНОЙ ПОЛИТИКИ. 1716-1726 ГГ., ПУБЛ. Д.О. СЕРОВА, ИСТОРИЧЕСКИЙ АРХИВ, № 6, 2000, С. 202.

7. СМ. СТ. 10-12 гл. 7-й, СТ. 5-9, 12, 16, 144, 146 и 170 гл. 10-й, СТ. 2 гл. 12-й, СТ.

83 ГЛ. 21-Й УЛОЖЕНИЯ (А.Г. МАНЬКОВ, РЕД., РОССИЙСКОЕ ЗАКОНОДАТЕЛЬСТВО Х-ХХ ВВ., Т. 3, М. : ЮРИДИЧЕСКАЯ ЛИТЕРАТУРА, 1985, С. 94, 102-104, 124, 157, 244).

8. ИСХОДЯ ИЗ КОНТЕКСТА, НАИБОЛЕЕ ТОЧНО ПЕРЕВЕСТИ ЭТУ ФРАЗУ НА РУССКИЙ ВОЗМОЖНО СЛЕДУЮЩИМ ОБРАЗОМ : « МНОЖЕСТВО ПОКАЗАТЕЛЕЙ УКАЗЫВАЕТ НА ТО, ЧТО ТОГДАШНЯЯ СУДЕБНАЯ СИСТЕМА ОБЕСПЕЧИВАЛА ВПОЛНЕ ПРИЕМЛЕМЫЙ УРОВЕНЬ ПРАВОСУДИЯ».

\section{AUTEURS}

\section{ДМИТРИЙ СЕРОВ}

Novosibirsk State University of Economics and Management 